\title{
Associations between length of stay and municipal characteristics for a large Norwegian stroke unit
}

Mathias Barra ( $\triangle$ mathias.barra@ahus.no)

Akershus Universitetssykehus HF https://orcid.org/0000-0002-0022-4042

Fredrik A. Dahl

Akershus Universitetssykehus HF

Kashif Waqar Faiz

Akershus Universitetssykehus HF

Hilde Lurås

Akershus Universitetssykehus HF

Research article

Keywords: hospital length of stay, model selection, regression modeling, econometrics, stroke unit, transfer of care

Posted Date: May 18th, 2020

DOI: https://doi.org/10.21203/rs.3.rs-27834/v1

License: (1) (1) This work is licensed under a Creative Commons Attribution 4.0 International License.

Read Full License 


\title{
Associations between length of stay and municipal characteristics for a large Norwegian stroke unit
}

\author{
Mathias Barra ${ }^{*}$, Fredrik A Dahl, Kashif W Faiz and Hilde Lurås
}

\footnotetext{
${ }^{*}$ Correspondence:

mathias.barra@ahus.no

$\mathrm{H} \varnothing \mathrm{KH}$ - The Health Services

Research Unit, Akershus

universitetssykehus HF, 1478

Lorenskog, Norway

Full list of author information is available at the end of the article

${ }^{\dagger}$ Corresponding author
}

\begin{abstract}
Background Determinants of length of stay (LOS) on hospital wards is important in many planning, policy, and decision problems. Furthermore, LOS is an important parameter for cost containment, and health managers maintain a strong focus on monitoring and reducing hospital LOS. In addition to being costly, excess LOS also expose the patient to unnecessary risk of in-hospital complications.

Methods We analysed a data set of $N=1922$ admissions to the stroke unit (SU) at Akershus University Hospital between February 2012 and March 2013, which contains high quality variables pertaining to each stay, including patient characteristics and municipality residency. We explored regression models with (log-)LOS as the dependent variable, economic and demographic characteristics of the patients' municipalities as the main predictors, controlling for several patient and admission parameters, like sex, age, and time of arrival.
\end{abstract}

Results The analyses showed that the municipality variables 'share of inhabitants below the age of 67 years who are female' and 'nurse full time equivalents per 1000 inhabitants' were negatively associated with LOS.

Conclusions Although a regression model cannot prove causal relations, a reasonable interpretation is that the given predictors capture some aspects of a municipality's capacity for home care, which makes it possible for the SU to discharge patients relatively early.

Keywords: hospital length of stay; model selection; regression modeling; econometrics; stroke unit; transfer of care 


\section{Background}

Determinants of length of stay (LOS) on hospital wards is important in many scheduling, policy, and decision problems. Furthermore, LOS is an important parameter for cost containment, $[1,2]$ and health managers maintain a strong focus on monitoring and reducing excess hospital LOS. In addition to being costly, excess LOS exposes the patient to unnecessary risk of in-hospital complications. [3] A temporal trend towards reduced mean LOS for somatic admissions is commonly observed in developed countries,[4] and has been documented by several studies.[5, 6]

The Norwegian health care system is divided into the primary health care and the specialist health services. Almost all Norwegian hospitals, - where the stroke units (SUs) are located - are public and financed by taxation, as is the primary health care in the municipalities. However, specialist health services are financed directly by the Ministry of Health and Care Services, while the delivery of primary health services and care, including post discharge care, is mainly the responsibility of the municipal health services (with financial support from the state.)

The decision to discharge a patient from a hospital ward, i.e. when treatment is deemed completed, is at the discretion of the treating physician. The physician can not, however, dictate the follow-up the patient is to have in the municipality. The hospital describe the patient's symptoms and level of function, and the municipality have the freedom and the responsibility to decide the level of further treatment and care.

The timely discharge from hospitals into lower-level care has in Norway often been seen in conjunction with the $\mathrm{LEON}^{[1]}$-principle. This means that incentives are being set up to facilitate and promote the transfer of care for inpatients to the primary health services in the home municipality of the patient.[7, 8] Most notably, the Coordination Reform ${ }^{[2]}$ that was implemented in 2012 has sought to set up the right balance between 'carrot and stick' so that health care professionals from specialist and primary health care would together ensure that adequate care can be provided at home for the patient; whether 'at home' means early supported discharge, a short- or long-term stay in an institution, or any other type of medical attention necessary for their continued recovery and care.[9]

If the municipality is not able to accept the patient within the discharge date set by the treating hospital physician, a daily fine is initiated until the patient is transferred to the municipality. The discharge is typically preceded by a discharge meeting with the patient and the next of kin. The communication with the municipality is usually written, unless the care givers in the municipality need additional information or want to assess the patient by themselves. In such cases, the municipal health services will visit the patient at the hospital before discharge, and initiate appropriate measures according to the assessment.

There is a vast literature on predicting LOS, spanning several methodological disciplines, [10] and it is outside the scope of this article to review the literature beyond mentioning a few relevant studies.

[1]'laveste effektive omsorgsnivå'; that is, lowest efficient level of care

${ }^{[2]}$ Samhandlingsreformen 
In a recent systematic review by Lu et al. which considers mainly regression modelling of LOS, the authors categorise the predictors used by researcher into conceptual groups: Social/Family, Patients characteristics, Healthcare System Characteristics, and Clinical Caregiver Characteristics in a conceptual framework for LOS modelling.[11, Figure 2, p. 363] The most commonly identified predictors are age, sex, and various comorbidity indices. In addition, a range of variables spanning hospital type, regions, urban/rural setting, the patients' social status (e.g. living alone) or different disease groups, have been investigated. Both these reviews concludes that the predictive value of LOS-models remain limited, $[11,10]$ that LOS is highly complex, and Lu et al.'s first recommendation is 'to explore the extent to which [non-patient] characteristics explain variation in LOS.'[11, p. 362] Nevertheless, there are few studies that focus on associations between municipal characteristics (MC's) - such as share of elderly or available nurses - and LOS, and this constitutes an important gap in the current knowledge on determinants of LOS.

It is also plausible that MC's should matter. One of the few studies we identified is Kjekshus' 2005 study, [12] which provided some evidence for an effect of municipal spending on primary health services on LOS. ${ }^{[3]}$ Another example is Holmås et al.'s 2013 study of LOS in which municipal health care expenditure variables were included, and which demonstrated a negative association between municipal spending on care services and LOS.[13]

A recent Stackelberg-game model proposed by Kverndokk \& Melberg also suggests that available resources influence LOS: faced with the decision of timing the transfer of care, the municipal health services will tend to delay the discharge from hospital when the costs ${ }^{[4]}$ are out-weighted by the benefits to the patient.[14] This could for example happen if supply of health care personnel or nursing home beds is low, but the health expenditure budget is not exhausted.

There have also been several studies on the role of health care professionals in the municipal services for the transfer of care; Tjerbo \& Kjekshus described GPs' perceptions of coordinating care,[15] while Ulin et al. have studied the effect of good communication between hospitals and municipal care services in Sweden.[16]

Furthermore, the individual resources and informal caregiver networks of a patient could influence a decision to expedite or delay the discharge of a patient.[17, 18, 19, 20]

${ }^{[3]}$ The full picture is somewhat more complicated, and Kjekshus' models and data are inconclusive viewed together; the paper contains a good discussion of possible mechanisms through which the regression results can be interpreted.

${ }^{[4]}$ Norwegian hospitals are reimbursed by the home municipality's primary health services for patients who are declared ready for discharge, but where an adequate service in the home municipality is not in place, resulting in an extended stay. 
In this study we make a modest step towards addressing this issue by undertaking an exploratory analysis of LOS-data from the 29 bed comprehensive stroke unit $(S U)^{[5]}$ at Akershus University Hospital (Ahus).

Although the data is from an SU, in this work we focus on the SU-patient, not the stroke patient. We therefore include patients with so-called stroke mimics - patients admitted with suspected stroke, but with a non-stroke diagnosis on discharge - as well as the patients with a transient ischemic attack in our data. However, patients admitted to an SU can be expected to be fairly heterogeneous in case-mix,[22] and a large share of the patients will be both elderly and frail. Hence, the LOS of these patients will likely be affected by the home municipality's care services capacity when treatment on the SU is completed.[16, 23, 24]

The paper proceeds as follows: In Section we introduce the data set with its variables and describe the steps of our regression modelling. In the first step we build a baseline model that uses patient characteristics and predictors related to the time of admission. In the second step we include the patient's municipality as a fixed effect in the model, and in the third step we substitute these fixed effects with characteristics of the patient's municipality. Note that the rationale for the second step was not to evaluate the impact of each municipality per se, but to test the predictive value of municipality related information in general. Since this test turned out positive, we proceeded to step three. Step three was the main aim of the analysis: to identify municipal characteristics that are associated with LOS, and to consider the case for a causal mechanism on LOS from the MC's found to have an association with LOS. Section 0.1 gives descriptive statistics and the results of the different regression models. In Section 0.1 we discuss our findings, and Section 0.1 concludes the article.

\section{Methods}

\subsection{Study setting}

The data set analysed is comprised of all $N=1922$ admissions to the SU at Ahus between February $15^{\text {th }} 2012$ and March $15^{\text {th }} 2013$. The admissions were consecutively registered, and include all admissions that resulted in a stroke or transient ischemic attack diagnosis as well as stroke mimics: admissions for suspected acute stroke that turned out to be caused by other morbidity.[22] These data were collected under the NOR-SPOT-project which collected data on a cohort of $2052 \mathrm{SU}$ admissions. Here we excluded patients that were either transferred from or to another ward or hospital (since their length of stay would be expectedly atypical), or those patients that did not belong to Ahus' catchment area (since these patients do not reside in any of the municipalities studied.) All

${ }^{[5]} \mathrm{A}$ stroke unit is a specialised ward with multidisciplinary staff for the diagnosing, treatment, and early rehabilitation of acute stroke; see e.g. Labberton et al. for a thorough discussion and definition of comprehensive stroke units. [21] 
patients resident in Ahus' catchment area are treated at Ahus unless they experience a stroke while traveling. In general, this holds of all acute medical emergencies that require hospitalisation in Norway: hospital of admission is dictated by geographical location of the patient. Ahus catchment area accounts for about $10 \%$ of Norway's population, and has varied demographics and urbanisation.

\section{Variables}

The dependent variable in our analyses is (stroke unit) length of stay (LOS), measured in hours. We initially considered both LOS and the log-transformed variable LLOS.

We restricted the analysis of step one to a set of key variables that are known to impact LOS. These can broadly be subdivided into two categories: variables that pertain to the patient, and variables that pertain to the admission.

For example, LOS has been positively associated with age, female sex, and co-morbidity. Furthermore, it is obvious that different diagnoses are associated with different expected LOS, and this has been shown for this data elsewhere.[22] These are patient-specific variables, and were available in the NOR-SPOT data set. In Table 2 these variables' definitions are found in rows with 'Pat' in the category (Cat.) column.

Secondly, variables pertaining to the admission are listed with 'Adm' in the Cat.-column. These variables are included to control for factors that may be associated with LOS because of how hospitals are run. For example, most non-acute diagnostics are performed and analysed during day-time, and therefore it is to be expected that a patient who is admitted late in the afternoon will have a somewhat longer hospital stay than a patient who is admitted early in the morning. Similarly, many wards operate with reduced staffing during holidays or weekends, and this might impact on LOS. Variables included for model exploration in this study were a dummy DayAdm for daytime admissions; HolAdm for holiday admissions; Wkd and Mth for day-of-the-week and monthof-the-year respectively, included to control for weekly or seasonal variance in LOS; and also a variable EDLOS which contained the LOS in the emergency department prior to transfer to the SU, the concern being that a very brief, or a very extended stay in the emergency department could be a confounder for e.g. a crowded SU.

For the purpose of this study, a third category of variables were considered for step three. These variables characterise the municipality of residence of the patients, are sub categorised as either demographic (Dem), Cost, or full time equivalents (FTE) in Table 2. These variables were harvested with Statistics Norway's StatBank's API ${ }^{[6]}$, and were chosen to shed light on the following hypotheses:

Hypothesis 1 Municipal spending on health care can influence the ability to receive patients with complex needs, and thus impact the expected LOS. We suspected that the expenditure data could

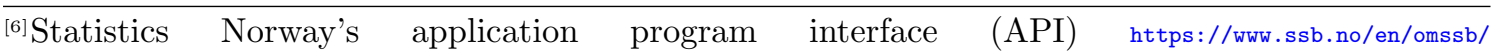

tjenester-og-verktoy/api 
either function as a flag where high expenditure is associated with a high number of care receivers and a relatively low flexibility in resource allocation, thus making it harder to effect the timely transfer of patients to municipal care following stroke, or, that high expenditure could impact the timely transfer of care in a positive way by indicating high capacity. That is, high expenditure could be forced upon the municipality by high demand, or it could be a chosen priority. We therefore obtained available data on municipal expenditure for health care purposes, broken down by cost groups. These variables are named according to the following convention: each cost group $(<\mathrm{CG}>)$ give a variable $\mathrm{CO}<\mathrm{CG}>/$ Cap with unit $1000 \mathrm{NOK}$ per capita (labeled 'Cost' in Table 2).

Hypothesis 2 The number of care-staff resources employed by the municipality could impact the municipality's ability to accept the transfer of care. Hence, we wanted to investigate if fewer full time equivalents is associated with prolonged LOS. Available municipal full time equivalents (FTE's) of key categories of health care professionals were therefore harvested, these are labelled 'FTE' in the Cat.-column, broken down by health personnel groups. These variables are named according to the following convention: each health personnel group $(<\mathrm{HPG}>$ ) give a variable $<$ HPG $>/$ Cap with unit FTE's per 1000 capita (labeled 'FTE' in Table 2).

Hypothesis 3 The 'resourcefulness' of patients could impact LOS. Median income, viewed as a proxy for expected resourcefulness of the typical patient in a municipality were therefore collected, as a highly resourceful population could possibly absorb some of the demand for post-discharge care needs. In a similar fashion, a populous municipality could enjoy economies of scale; a relative surplus of younger females, could serve as a substitute for municipal services; or a relatively high share of elderly could strain available municipal capacity for receiving discharged patients. Therefore, variables for demographic composition was also investigated: Pop the number of inhabitants, Pop $(67+)$ Share the fraction of inhabitants older than 67 years of age, and the Fem(66-)Share sexratio of the municipalities' younger population. The link, if any, between female share and LOS is the most indirect one. We do not hypothesise that increasing the number of younger women in a municipality will impact LOS. Instead, we think that in a municipality with more younger women, the probability that a typical patient has a female caregiver is higher, [25] and that for those patients, LOS may be shorter. Such effects could be discernible in a large data set like ours. These variables are labeled with 'Dem' in Table 2.

Lastly, the variable AoD is a dummy for Alive on Discharge, and belong among the patient variables. Since our main hypothesis is that LOS is associated with municipal factors to the extent that they mediate the municipalities' ability to effect the transfer of care, we considered it prudent to control for whether or not the patient survived the acute phase: deceased patients are discharged at the time of death, regardless of any other factors.

All the variables, their sources, and definitions are displayed in Table 2

The Statistics Norway-data are available on a yearly basis, and we used data for the year of the $S U$-admission in each row: admissions from 2012 were matched with municipal data for 2012, and 
similarly for admissions from 2013. The pecuniary data is adjusted for inflation using the Norwegian KPI-index, ${ }^{[7]}$ a measure of domestic inflation.

Referring to Table 2, e.g. the 'nurse-FTE-per-capita'-variable contains

$$
(\text { Nur } / \text { Cap })_{j}:=\frac{\text { number of nurse FTE's in municipality } j}{\operatorname{Pop}_{j}} \times 10^{-3}
$$

while the 'cost of care-provided-in-homes-per-capita'-variable is defined by

$$
(\text { COCAHO } / \text { Cap })_{j}:=\frac{\text { costs towards care provided at patients' homes in municipality } j}{\operatorname{Pop}_{j}} \times 10^{3} \text {. }
$$

\section{Estimation strategy}

We computed descriptive statistics for the data set, both across patients and municipalities. Inspection of the LOS-distribution revealed highly non-normal data. A log-transformation resulted in a left-skewed distribution, which upon initial regression test-specifications yielded acceptable behavior of the residuals as judged by inspection of the QQ-plots. We chose to continue our analyses with respect to the log-transformed data. This induces a semi-elasticity-interpretation on the regression coefficients; see section 0.1 for details. The resulting log-LOS distribution is shown in Figure 1 in the Appendix.

An important issue to consider is the ratio $n_{\mathrm{Mun}}: n_{\mathrm{MR}}$ of the number of municipalities $\left(n_{\mathrm{Mun}}=24\right)$ to the number of municipal regressors $\left(n_{\mathrm{MR}}\right)$ included in the regression specification; because each municipal variable is constant over patients from the same home municipality and discharge year, it is important to mitigate over-specification. Therefore, we specified our models in several steps:

We first inspected the bivariate associations between the patient- and admission variables and logLOS. Based on previous literature, in-group medical expertise ${ }^{[8]}$, and inspection of the bivariate plots, we selected a base specification with regressors among these variables. We then performed a full-factor exploration of the specification space, and compared the fits by Akaike's Information Criterion (AIC), Bayesian Information Criterion (BIC), and the root mean squared error (RMSE) obtained from a leave-one-out cross validation (LOOCV) routine.

The information criteria employed (AIC and BIC) are used to compare model fit when the set of regressors are mutually non-overlapping, and provides guidance on variable selection based on likelihood analysis. [26, 27, 28] For AIC and BIC a lower value indicates a higher likelihood of the data given the specification. These fit measures adjust for the number of included regressors, and BIC impose a higher penalty for additional regressors than do the AIC.

${ }^{[7]}$ https://www.ssb.no/en/priser-og-prisindekser/statistikker/kpi

${ }^{[8]}$ The third author is a neurologist with extensive clinical experience from the SU where the data was collected, and from hospital management roles. 
LOOCV is a model evaluation technique that is based on sequential estimations of the model. The model is estimated on a data set where a single data point is left out, and its prediction is measured on that data point. This process is repeated for all the data points, and the average prediction error is calculated. The method gives a good estimate of the model's prediction error on unseen data and therefore provides a useful criterion for model selection.[29, 30]

This model selection results in a specification $\operatorname{LLOS}_{i}=\beta \mathrm{Base}_{i}+\varepsilon_{i}$ where the $\mathrm{Base}_{i}$ regressors are chosen from the categories patient (Pat) and admission (Adm) variables in Table 2.

Next, and prior to introducing specific municipal characteristics $\mathrm{MC}_{\mathrm{k}}$ 's, we added municipal fixed effects (MFE) to the base model to establish whether the inclusion of municipal variables seemed reasonable: $\operatorname{LLOS}_{i}=\boldsymbol{\beta}$ Base $_{i}+\boldsymbol{\delta}_{j(i)}+\varepsilon_{i}$ where $\boldsymbol{\delta}_{j}$ is a vector of dummy variables for municipality, and $j(i)$ picks out the home municipality ${ }^{[9]} j$ of patient $i$.

The idea is that if municipal characteristics - like median income or costs towards care in institutions - do in fact associate with LOS, then a specification with MFEs would detect the betweenmunicipality heterogeneity. For this second step we considered AIC, BIC, and $\mathrm{R}^{2}$ for guidance, and the MFE fit was subjected to a standard ANOVA analysis.

Conditional on the evidence for municipal effects provided by the second step, we planned to fit one model

$$
\operatorname{LLOS}_{i}=\beta \text { Base }_{i}+\mu_{k} \mathrm{MC}_{k, j(i)}+\varepsilon_{i}
$$

for each $\mathrm{MC}_{k}$, and to considered whether $\mathrm{R}^{2}$, $\mathrm{AIC}$, or BIC improved upon their inclusion. In this notation $\mathrm{MC}_{k, j(i)}$ denotes the $k^{\text {th }}$ municipal variable's value for $j(i)$ as above.

Based on the results from these analyses, we conducted a second full-factor search through the specification space constituted by the set of base regressors together with the most promising MC's.

Furthermore, as a sensitivity analysis, we fitted the final specification to several subsets of the full data set. In particular, we wanted to investigate the following four sources of potential bias:

Sensitivity analysis 1) In 2011, Ahus' catchment area was expanded to include the three Oslo districts Alna, Grorud, and Stovner; prior to this Ahus' catchment area consisted of 21 municipalities of Akershus county (see also map in Table 4) As the data set investigated in this work pertains to 2012 - 2013 it seemed diligent to investigate whether a separate Oslo-effect could introduce missingvariable bias. Moreover, as pointed out above, the FTE data was only available at municipal level, so that Oslo's mean value has been used for these three districts in the full analysis. Hence, if the number of FTE's within any one care staff-category was very different from the other municipalities, one could suspect that a seeming association was due not to the number of FTE's, but to an Oslo effect. For example, it seems reasonable that newly added geographic areas could experience more

${ }^{[9]}$ In fact, $\delta_{j(i)}$ also depends on the year of patent $i$ 's admission, but we have suppressed this from the notation. 
friction during the discharge process of patients to municipal care, while routines and professional networks are still developing.

Sensitivity analysis 2) Furthermore, for two municipalities ${ }^{[10]}$, FTE-data was not available from Statistics Norway; We imputed zero FTE's where data was missing, and therefore repeated the model fitting omitting data from these municipalities.

Sensitivity analysis 3) We also fitted a specification with only the retained MC's, so as to assess whether the identified effects were stable also when otherwise informative patient- and admission variables were kept out.

Sensitivity analysis 4) Lastly, in order to assess to what extent identified associations between MC's and patient LOS could be construed as causal, we tested the final specification on the two data sets obtained by splitting according to whether the patient were discharged alive or not. The idea is that an MC's main causal effect should be to facilitate mechanisms for the timely transfer of care for a patient from the hospital; and deceased patients cannot benefit from such mechanisms.

All analyses were performed with the statistical software package $\mathrm{R}$ within the RStudio environment; model estimation used the standard lm-function (OLS), graphical output was rendered with plotly and stargazer.[31, 32, 33, 34]

\section{Results}

\section{Descriptive statistics - admissions}

The data set contains information on $N=1922$ included admissions of patients from Ahus' catchment area (82 admissions from non-Ahus patients, and 48 in- or between-hospital transfers were excluded from the full data set of 2052 admissions); $51.8 \%$ were male; $62.0 \%$ had a daytime admission $($ DayAdm $=1) ; 7.3 \%$ had a holiday admission $($ HolAdm $=1)$. Ischemic strokes accounted for $37.6 \%$; haemorrhagic strokes for $6.9 \%$; transient ischemic attacks for $17.1 \%$, and $38.4 \%$ were stroke mimics; 99 admissions $(5.2 \%)$ resulted in an in-hospital fatality $(\mathrm{AoD}=0)$.

Patients in our sample had a mean age of 70.5 years, and the typical number of comorbidities were 1 (median), with an IQR of $0-2$. LOS at the ED was typically 3 hours (median). The LOS and log-LOS distributions were discussed in Section 0.1, and see also Table 3 for further descriptive statistics for the continuous variables.

The most interesting bi-variate analysis was noting an (expected) quadratic relationship between $\log$-LOS and the number of previous conditions. This relationship informed the addition of a quadratic term for $\Sigma P C$ in the model specifications.

${ }^{[10]}$ Nesodden and Rømskog 


\section{Descriptive statistics - municipalities}

There are 24 municipalities, as shown on the map in Table 4, and they displayed a sufficient variation in the MC's to possibly isolate any associations with SU LOS. The mean LOS for patients from the different municipalities is also tabulated in Table 4.

The descriptive statistics are present in the Tables 9,10 , and 11, and are deferred to the appendix.

Regression analysis and model selection

The LOS-distribution was severely right skewed, so a log-transformed variable was used in the analyses; see Figure 1 in the Appendix. This means that the interpretation of regression coefficients is a semi-elasticity: a unit increase in an independent variable $X$ with coefficient $\beta$ predicts an increase of $100\left(e^{\beta}-1\right) \%$ in LOS. This transformation led to a much better distribution of residuals, and is commonly employed in the LOS-literature.[11]

While the number of observations in the data set is sufficiently high to test specifications with 10 20 regressors, there are only 24 municipalities/districts. This means that some caution is warranted when testing specifications that include the $\mathrm{MC}_{i}$ 's. We therefore split the model selection phase into several steps: In the first, we considered models with only patient and admission variables, and started with the specification $\operatorname{LLOS}_{i}=\alpha+\boldsymbol{\beta} \boldsymbol{X}_{i}+\varepsilon_{i}$ where $\boldsymbol{X}=\left(\mathrm{P}_{1}, \ldots, \mathrm{P}_{5}, \mathrm{~A}_{1}, \ldots, \mathrm{A}_{6}\right)$; see Table 2 .

We next performed a full-factor search, considering the AIC, BIC, and RMSE for a LOOCV for all combinations of these regressors: The results (the best candidates are displayed in Table 7; in the appendix) were then judged by overall merit.

We choose the six-variable model indicated by the lowest RMSE, since the seven-variable specification suggested by the lowest AIC was only marginally better in terms of AIC, while the three-variable specification suggested by lowest BIC had notably higher RMSE from the LOOCV.

This yielded the following base-model (see also Table 5):

Base model :=

$\operatorname{LLOS}_{i}=\alpha+\boldsymbol{\beta} \underbrace{\left(\mathrm{STR}_{i}+\Sigma \mathrm{PC}_{i}+\mathrm{AoD}_{i}+\mathrm{LOS}_{\mathrm{ED} i}+\operatorname{HolAdm}_{i}+\mathrm{DayAdm}_{i}\right)}_{\text {Base }}+\varepsilon_{i}=$ $\operatorname{LLOS}_{i}=\beta$ Base $_{i}+\varepsilon_{i}$.

To this base specification we next fitted a specification with MFE's as a test of whether further exploration of specific MC's seemed reasonable.

The regression results - from the full model, the reduced base model, and the base model with MFEs - are are presented in Table 5 together with several fit-statistics. 
We note that the base model's coefficients appear stable; they are not perturbed much by adding or removing regressors. The addition of the MFE's does not seem to improve the fit much, in particular if one consults the AIC or BIC for guidance. However, looking to the (adjusted) $\mathrm{R}^{2}$ there is a slight improvement in explained variance when MFE's are added, the ANOVA analysis yields an overall $p$-value of 0.01 for the MFE's, and the coefficient estimates for the base-regressors are similar in the MFE-model and in the base model.

Based on the above, we decided to continue testing the specific $\mathrm{MC}_{k}$ 's. The coefficients on the $\mathrm{MC}_{k}$ 's when fitting the data to specifications (for each $k=1, \ldots, 15$ )

$$
\operatorname{LLOS}_{i}=\beta \mathrm{Base}_{i}+\mu_{k} \mathrm{MC}_{k, j(i)}+\varepsilon_{i}
$$

are presented in Table 1, alongside their standard errors, $p$-values, and the differences in AIC and BIC between the base model and the model augmented with the $\mathrm{MC}_{k}$ ( $\triangle \mathrm{AIC}$ and $\triangle \mathrm{BIC}$ respectively.) The four variables Nur/Cap, GerNur/Cap, COCAIN/Cap, and Fem(66-)Share were selected for the final model selection step as these each improved AIC by a full point or more, none increased BIC by more than five, and each had an associated $p$-values below 0.1 .

The final step consisted of performing a second full-factor specification analysis, testing all possible specifications over the set of base-regressors and these four MC's. The results (Table 8; in Appendix) showed that both the LOOCV routine, and the AIC agreed on an eight-variable specification

$$
\operatorname{LLOS}_{I}=\boldsymbol{\beta B a s e}_{i}+\mu_{9} \text { Nur } / \text { Cap }_{j(i)}+\mu_{3} \text { Fem }_{(66-)} \text { Share }_{j(i)}+\varepsilon_{i}
$$

and the result from fitting this specification to our data set is shown in Table 6 . The four variable specification pointed to by the BIC is the same as is seen when selecting the base model. There is a substantial improvement in AIC, but only a modest improvement in cross-validation performance.

The log-linear specification yields coefficient estimates $\beta$ that have a semi-elasticity interpretation: a unit change in the variable effects a $\exp (\beta)-1$ percent change in the untransformed dependent variable (LOS). The patient-specific coefficients overall tells the expected story: compared to the reference group of ischemic strokes, the stroke mimics and the transient ischemic attack patients have an approximately $50.7 \%$ and $48.1 \%$ shorter LOS, respectively. The case fatalities have $47.5 \%$ shorter LOS on the SU than patients who are discharged alive. Furthermore, for each additional previous condition, estimated LOS is increases by $2.9 \%$. The admission-specific variables tell us that each extra hour in the emergency department before transfer to the $\mathrm{SU}$ is expected to increase the SU-LOS with $0.2 \%$, being admitted $7 \mathrm{am}-7 \mathrm{pm}$ leads to a $16.8 \%$ longer LOS, and discharges for holiday admissions are delayed: LOS is expected to increase by approximately $21.3 \%$ for these patients.

In interpreting the coefficients for share of females and for nurse FTEs per capita, we must be mindful of the units. The Fem(66-)Share variable is given as a percentage, and the IQR for the 
municipalities in our sample is $48.7 \%-49.4 \%$. This means that an increase of the share of the population below 67 years of age by one percentage point, is estimated to reduce LOS by $\exp (0.01$. $-8.57)-1=0.082$, that is, an $8.2 \%$ decrease in expected LOS. The variable for nurse FTEs has unit nurse FTEs per 1000 inhabitants, the IQR in our sample is 2.7-3.2 nurse FTEs per 1000 inhabitants. A reasonable change can then be taken as, say, 0.1 additional nurse FTEs per 1000 inhabitants, and our model predicts that this would lead to a decrease in expected LOS of $\exp (0.1 \cdot-0.109)-1=1.1 \%$.

\section{Sensitivity analyses}

All sensitivity analyses showed that the final specification was relatively stable over the various subsets of the data, with one notable exception: when the model was fitted to only those patients who were not discharged alive, none of the regressors were significant except ICH $(p<0.001)$ which was negative. ${ }^{[11]}$ Reassuringly, restricting the data set to patients that were discharged alive, no significant perturbation of the fit was observed; see Table 12 in the appendix for the various models.

\section{Discussion}

We have found moderately strong evidence for the hypothesis that the number of available nurses within the municipal health care system is associated with the timely transfer of care from the SU. We would even venture to conjecture that this might constitute a causal mechanism.

Our results can be seen as corroborating previous results by Kjekshus,[12] Holmås et al.,[13] and Gautun \& Syse.[8] who assert that 'variation in LOS between hospitals may be explained mainly by the capacity of primary health care providers. 'While our findings do not support nurse FTE's as a main driver of LOS, our study corroborates FTE's as a contributing factor.

Surprisingly, none of the cost-variables had a discernible effect on LOS that survived the model selection phase. This contrasts the findings from Kjekshus and from Holmås et al.[12, 13] Clearly, a higher nurse FTE per capita will impact municipal costs; in particular in Norway where wages account for a substantial fraction of health allocations. However, in our analyses, there was no direct effect of municipal health expenditure on the LOS of their patients, that was not better accounted for by the FTE-variables. This suggests that the mechanism through which increased expenditure lead to shorter LOS, could mainly go though municipally employed nurses.

Our results could be caused by unmeasured confounders; e.g. a higher nurse density in a municipality could be a response to an elevated demand, which, in turn, could also impact the municipality's ability to receive discharged patients. However, of the cost variables, only costs at care institutions (COCAIN/Cap) was associated with LOS (Appendix; Table 1), and in the models where both nurse ${ }^{[11]}$ One interpretation is simply that municipal characteristics do not associate with time to death, and that patients with haemorrhagic stroke who die during their hospital stay tend to die faster than other SU patients. 
FTE's and costs were included, the fit statistics consistently pointed to Nur/Cap as the most important predictor (Appendix; Table 8). If the effect of Nur/Cap is, in fact, entirely spurious, we would have expected the confounder to also be detectable via some of the other variables studied here.

It is noteworthy that neither sex nor age were significantly associated with LOS. These variables were left out after choosing the base specification, but we reintroduced sex and age to the final specification as a sensitivity analysis without any change of the conclusion ( $p=0.66$ and $p=0.49$ respectively.) We did observe a strongly significant correlation (Pearson's $r=0.31, p<0.0001$ ) between number of diagnoses and age. Although age has been shown to predict LOS in other studies,[11] it was the number of diagnoses that best explained the variance in our data, suggesting that it is mostly the increased morbidity associated with age, and not age in itself, that drives increased LOS.

The link to female share hinted to by the regression results - i.e. that the more women per capita in the age bracket $0-66$ there are, the earlier patients are discharged - is novel and slightly surprising. It has been shown that female caregivers tend to supply more informal care than their male counterparts, and it is not inconceivable that a larger total supply of informal care in a municipality could translate into a measurable impact on LOS. Here, we can clearly not conclude, but the effect size and $p$-value remained stable throughout the sensitivity analyses. We do not believe that this is a direct effect, but perhaps this finding reflects an elevated likelihood of facilitated transfer of care in municipalities with (relatively) more younger women, either because this is an confounder, or because it is a mediator for better access to home-care.[35, 36, 37, 38] As remarked upon when detailing our hypothesis 3 , this is likely a proxy for a patient-specific effect.[25]

It is obvious that the transfer of care of elderly and multi-morbid patients into municipal care depends on many factors. That availability of nurses and informal caregivers -captured by the Nur/Cap and - to some extent - Fem(66-)share variables is quite possible, given the large number of studies that demonstrate the importance of these factors.[35, 36, 37, 39, 40]

Municipalities in our data set vary substantially, both with respect to population size, demographic composition, and centrality. Since all patients in our data were discharged from the same hospital, the observed differences are not due to between-hospital variation. A strength of this study is therefore the large catchment area of Ahus, and the unselected patient sample.

On the other hand, we only included data from two different years (2012 and 2013) in our analyses, and therefore the number of observations is relatively low for the MC's with only 24 municipalities. Another important limitation of our study is that we did not include data on pre-hospital or arrival level of function (e.g. mRS or Barthel score [41, 42]) as these were incomplete for many transient ischemic attacks and stroke mimics patients. Pre-hospital function of patients is associated with a number of outcomes, that could influence a decision to discharge a patient, and future studies with better individual level data should focus on the patients' network of caregivers and their functional levels in order to better understand this phenomenon. 
As for the predictive value of our models, this study confirm that LOS is notoriously hard to predict.[11, 43] All the regression models produced adjusted $\mathrm{R}^{2} \leq 0.14$, meaning that less than $14 \%$ of the variance in the observed LOS is explained by our models. The addition of MC's to the base model increased the amount of explained variance by a mere $3.4 \%$ (from about $12.96 \%$ to $13.41 \%$ ). In our data, most of the variation in LOS is thus explained by patient characteristics, consistent with the literature.[11] Furthermore, the $\mathrm{R}^{2}$ and the LOOCV's RMSE of the model selected by BIC is quite similar to the values reported for the selected final model.

The implication of this is that despite fairly low $p$-values, what we have found are not factors that are useful for e.g. SU capacity planning. Instead our findings may point to testable conjectures about causal mechanisms that influence (SU) LOS that can be researched further. That is, we found evidence that is consistent with, but not proof of, such mechanisms.

\section{Conclusion}

The presented analysis shows that although several predictors are statistically significant for LOS in the stroke unit of Ahus, the model's residual variance is too high for prediction purposes. However, we found support for the hypothesis that characteristics of municipal health care services may have a causal impact on LOS; specifically that an increasing number of nurse FTE's may facilitate more timely transfer into municipal care. Furthermore, a higher fraction of young female inhabitants might have a similar effect. Our results must be interpreted with caution, and further studies are necessary to validate these findings.

\section{Abbreviations}

$\mathrm{LOS}=$ length of stay; $\mathrm{SU}=$ stroke unit; $\mathrm{GP}=$ general practitioner; FTE $=$ full time equivalent; API application program interface; AIC = Akaike information criterion; $\mathrm{BIC}=$ Bayesian infomration criterion; RMSE = root mean squared error; LOOCV = leave one out cross validation; OLS = ordinary least squares; NLOD = Norwegian license for open government data; MFE = municipal fixed effect; ANOVA = analysis of variance

\section{Declarations}

\section{Ethics approval and consent to participate}

NOR-SPOT is an in-hospital project investigating the paths of treatment without any deviation from standard practices and has been classified as a quality assurance project by the Regional Ethics Committee. Therefore, and in accordance with the Regional Ethics Committee's recommendation, ethical approval was sought with the Privacy Ombudsman at Akershus universitetssykehusl HF (approval no. 11-076).

\section{Consent for Publication}

All authors declare their consent for publication. 


\section{Availability of data and material}

Data on the stroke unit admissions is not available due to privacy restriction. The data harvested from Statistics Norway is open domain data available for non-commercial use under the NLODlicense. ${ }^{[12]}$. The $\mathrm{R}$ code will be made available from the corresponding author upon reasonable request.

\section{Competing interests}

All authors declare no conflicts of interest nor competing interests.

\section{Funding}

Dr. M. Barra, Dr. F.A. Dahl, were partially funded by grant nos. 196454 and 237809 Dr. K.W. Faiz was paritally funded by grant no. 237809

\section{Authors' contributions}

MB collected data, designed, and performed the analyses, drafted the manuscript, and coordinated revisions. FAD designed the analyses, interpreted results, and revised the manuscript for content. KWF provided medical interpretations, interpreted results, and revised the manuscript for content. HL conceived the analyses, interpreted results, and revised the manuscript for content.

\section{Acknowledgements}

NA

\section{References}

1. S. K. Saxena, T. P. Ng, D. Yong, N. P. Fong, and K. Gerald. Total direct cost, length of hospital stay, institutional discharges and their determinants from rehabilitation settings in stroke patients. Acta Neurologica Scandinavica, 114(5):307-314, November 2006

2. Hoang V. Tran, Darleen Lessard, Mayra S. Tisminetzky, Jorge Yarzebski, Edgard A. Granillo, Joel M. Gore, and Robert Goldberg. Trends in Length of Hospital Stay and the Impact on Prognosis of Early Discharge After a First Uncomplicated Acute Myocardial Infarction. The American Journal of Cardiology; New York, 121(4):397-402, February 2018.

3. Aleen Clarke and Rebecca Rosen. Length of stay: How short should hospital care be? European Journal of Public Health, 11(2):166-170, June 2001

4. OECD. Health at a Glance 2017. OECD Indicators, OECD, paris, France, 2017.

5. Héctor Bueno, Joseph S. Ross, Yun Wang, Jersey Chen, María T. Vidán, Sharon-Lise T. Normand, Jeptha P. Curtis, Elizabeth E. Drye, Judith H. Lichtman, Patricia S. Keenan, Mikhail Kosiborod, and Harlan M. Krumholz. Trends in Length of Stay and Short-term Outcomes Among Medicare Patients Hospitalized for Heart Failure, 1993-2006. JAMA, 303(21):2141-2147, June 2010.

6. Jun Yin, Hilde Lurå s, Terje P. Hagen, and Fredrik A. Dahl. The effect of activity-based financing on hospital length of stay for elderly patients suffering from heart diseases in Norway. BMC Health Services Research, 13(1):172, May 2013.

7. M. Irene Puhr and Hilaire J. Thompson. The Use of Transitional Care Models in Patients With Stroke:. Journal of Neuroscience Nursing, 47(4):223-234, August 2015.

8. Heidi Gautun and Astri Syse. Earlier hospital discharge: a challenge for Norwegian municipalities. Nordic Journal of Social Research, 8, July 2017.

9. Lars C. Monkerud and Trond Tjerbo. The effects of the Norwegian Coordination Reform on the use of rehabilitation services: panel data analyses of service use, 2010 to 2013. BMC Health Services Research, 16(1):353, August 2016.

${ }^{[12]}$ Norwegian Licence for Open Government Data; see https://data.norge.no/nlod/en/2.0 
10. Aya Awad, Mohamed Bader-El-Den, and James McNicholas. Patient length of stay and mortality prediction: A survey. Health Services Management Research, 30(2):105-120, May 2017.

11. Mingshan Lu, Tolulope Sajobi, Kelsey Lucyk, Diane Lorenzetti, and Hude Quan. Systematic Review of Risk Adjustment Models of Hospital Length of Stay (LOS). Medical Care, 53(4):255-365, April 2015.

12. Lars Erik Kjekshus. Primary health care and hospital interactions: Effects for hospital length of stay. Scandinavian Journal of Public Health, 33(2):114-122, March 2005.

13. Tor Helge Holmås, Mohammad Kamrul Islam, and Egil Kjerstad. Between two beds: inappropriately delayed discharges from hospitals. International Journal of Health Care Finance and Economics, 13(3-4):201-217, December 2013.

14. Snorre Kverndokk and Hans Olav Melberg. Using Fees to Reduce Bed-Blocking: A Game between Hospitals and Care Providers. SSRN Scholarly Paper ID 2877111, Social Science Research Network, Rochester, NY, October 2016.

15. Trond Tjerbo and LarsErik Kjekshus. Coordinating health care: lessons from Norway. International Journal of Integrated Care, 5, November 2005

16. Kerstin Ulin, Lars-Eric Olsson, Axel Wolf, and Inger Ekman. Person-centred care - An approach that improves the discharge process. European Journal of Cardiovascular Nursing, 15(3):e19-e26, April 2016.

17. Lolita Jacob and Eileen Blechman Poletick. Systematic review: Predictors of successful transition to community-based care for adults with chronic care needs. Care Management Journals, 9(4):154-65, 2008. Copyright - Copyright Springer Publishing Company 2008; Last updated - 2018-10-06.

18. France M. Weaver and Bryce A. Weaver. Does availability of informal care within the household impact hospitalisation? Health Economics, Policy and Law, 9(1):71-93, 2014.

19. Tor Helge Holmås, Karin Monstad, and Darina Steskal. Hospital Readmission and Mortality Among Frail Elderly - The Importance of Spouse and Adult Children. Working paper, Stein Rokkan Centre for Social Studies, December 2015.

20. Audhild Høyem, Deede Gammon, Gro Berntsen, and Aslak Steinsbekk. Keeping one step ahead: A qualitative study among Norwegian health-care providers in hospitals involved in care coordination for patients with complex needs. International Journal of Care Coordination, 21(1-2):15-25, June 2018.

21. Angela Susan Labberton, Ole Morten R $\varnothing$ nning, Bente Thommessen, and Mathias Barra. Changes in survival and characteristics among older stroke unit patients—1994 versus 2012. Brain and Behavior, 0(0):e01175, November 2018.

22. Kashif Waqar Faiz, Angela Susan Labberton, Bente Thommessen, Ole Morten Rønning, Fredrik A. Dahl, and Mathias Barra. The Burden of Stroke Mimics: Present and Future Projections. Journal of Stroke and Cerebrovascular Diseases, 0(0), January 2018.

23. Peter Langhorne, Satu Baylan, and Early Supported Discharge Trialists. Early supported discharge services for people with acute stroke. Cochrane Database of Systematic Reviews, (7), 2017.

24. Janet Prvu Bettger, Sara B. Jones, Anna M. Kucharska-Newton, Janet K. Freburger, Sylvia W. Coleman, Laurie H. Mettam, Mysha E. Sissine, Sabina B. Gesell, Cheryl D. Bushnell, Pamela W. Duncan, and Wayne D. Rosamond. Meeting Medicare requirements for transitional care: Do stroke care and policy align? Neurology, page 10.1212/WNL.0000000000006921, January 2019.

25. Joukje Swinkels, Theo van Tilburg, Ellen Verbakel, and Marjolein Broese van Groenou. Explaining the Gender Gap in the Caregiving Burden of Partner Caregivers. The Journals of Gerontology: Series B, 74(2):309-317, January 2019. Publisher: Oxford Academic.

26. Robert E. Kass and Adrian E. Raftery. Bayes Factors. Journal of the American Statistical Association, 90(430):773-795, June 1995.

27. David Posada and Thomas R. Buckley. Model Selection and Model Averaging in Phylogenetics: Advantages of Akaike Information Criterion and Bayesian Approaches Over Likelihood Ratio Tests. Systematic Biology; Oxford, 53(5):793-808, October 2004.

28. Kenneth P. Burnham and David R. Anderson. Multimodel Inference: Understanding AIC and BIC in Model Selection. Sociological Methods \& Research, 33(2):261-304, 2004.

29. Bradley Efron and Gail Gong. A Leisurely Look at the Bootstrap, the Jackknife, and Cross-Validation. The American Statistician, 37(1):36-48, 1983.

30. Jerome Friedman, Trevor Hastie, and Robert Tibshirani. The Elements of Statistical Learning - Data Mining, Inference, and Prediction. Number 10 in Springer series in statistics. Springer New York, New York, USA, 2nd edition, 2008.

31. R Core Team. R: A Language and Environment for Statistical Computing, 2017.

32. RStudio Team. RStudio, 2016.

33. Carson Sievert, Toby Hocking, Scott Chamberlain, Karthik Ram, Marianne Corvellec, and Pedro Despouy. plotly: Create Interactive Web Graphics via 'plotly.js', July 2017.

34. Marek Hlavac. Stargazer: Well-Formatted Regression and Summary Statistics Tables, 2018.

35. Linda S. Noelker and David M. Bass. Home Care for Elderly Persons: Linkages Between Formal and Informal Caregivers. Journal of Gerontology, 44(2):S63-S70, March 1989.

36. Tamilyn Bakas, Joan K. Austin, Samantha L. Jessup, Linda S. Williams, and Marilyn T. Oberst. Time and Difficulty of Tasks Provided by Family Caregivers of Stroke Survivors. Journal of Neuroscience Nursing; Park Ridge, 36(2):95-106, April 2004.

37. Lui May H.L., Ross Fiona M., and Thompson David R. Supporting Family Caregivers in Stroke Care. Stroke, 36(11):2514-2522, November 2005.

38. Fiona Carmichael, Susan Charles, and Claire Hulme. Who will care? Employment participation and willingness to supply informal care. Journal of Health Economics, 29(1):182-190, 2010

39. Ragnhild Helles $\varnothing$ and May Solveig Fagermoen. Cultural diversity between hospital and community nurses: implications for continuity of care. International journal of integrated care, 10(1), 2010

40. Rose M. Olsen, Bjørg H. Østnor, Ingela Enmarker, and Ove Hellzén. Barriers to information exchange during older patients' transfer: nurses' experiences. Journal of Clinical Nursing, 22(19-20):2964-2973, 2013. 
1

41. Florence I. Mahoney and Dorothea W. Barthel. Functional evaluation: The Barthel Index: A simple index of independence useful in scoring improvement in the rehabilitation of the chronically ill. Maryland State Medical Journal, 14:61-65, 1965. Place: US Publisher: Medical and Chirurgical Faculty of the State of Maryland.

42. Jamie L. Banks and Charles A. Marotta. Outcomes validity and reliability of the modified Rankin scale: implications for stroke clinical trials: a literature review and synthesis. Stroke; a Journal of Cerebral Circulation, 38(3):1091-1096, March 2007.

43. M. M. Stecker, M. Stecker, and J. Falotico. Predictive model of length of stay and discharge destination in neuroscience admissions. Surgical Neurology International, 8(1):17, 2017.

44. Achim Zeileis. Econometric computing with HC and HAC covariance matrix estimators. Journal of Statistical Software, 11(10):1-17, 2004. 


\section{Supplementary Files}

This is a list of supplementary files associated with this preprint. Click to download.

- Appendices.pdf 